\title{
Description of ionophore-doped membranes with a blocked interface
}

\author{
Tarun M. Sanders ${ }^{\mathrm{a}}$, Matthew Myers ${ }^{\mathrm{b}, \mathrm{d}}$, Mohsen Asadnia ${ }^{\mathrm{a}, \mathrm{e}}$, Gilberto A. \\ Umana-Membreno $^{\mathrm{a}}$, Murray Baker ${ }^{\mathrm{b}}$, Neville Fowkes ${ }^{\mathrm{c}}$, Giacinta Parish ${ }^{\mathrm{a}, *}$, \\ Brett Nener ${ }^{\mathrm{a}}$ \\ ${ }^{a}$ School of Electrical, Electronic and Computer Engineering, The University of Western \\ Australia, 35 Stirling Hwy., Crawley, Western Australia 6009, Australia \\ ${ }^{b}$ School of Molecular Sciences, The University of Western Australia, 35 Stirling Hwy., \\ Crawley, Western Australia 6009, Australia \\ ${ }^{c}$ School of Mathematics and Statistics, The University of Western Australia, 35 Stirling \\ Hwy., Crawley, Western Australia 6009, Australia \\ ${ }^{d}$ CSIRO Energy Business Unit, Kensington, Western Australia 6151, Australia \\ ${ }^{e}$ Department of Engineering, Macquarie University, Sydney, New South Wales 2109,
} Australia

\begin{abstract}
Ion sensors with blocked interfaces, such as coated-wire electrodes (CWEs) and ion-selective field effect transistors (ISFETs), are increasingly being used as alternatives to traditional ion-selective electrodes (ISEs). This work provides insights into the behaviour of ionophore-doped membranes in solution, where one side completely blocks ion transfer, using the Nernst-PlanckPoisson (NPP) model, including reaction terms. Extensive investigations were conducted, demonstrating that the NPP model provides reasonable predictions, as well as insight into the interplay between interfacial kinetics, complexation, diffusion and electrical processes. Model validation is achieved through its application to and analysis of experimental results obtained for AlGaN/GaN ISFETs with ionophore-doped membranes.
\end{abstract}

Keywords:

Nernst-Planck-Poisson, Membranes, Ionophore, Electro-diffusion

* Corresponding author

Email address: giacinta.parish@uwa.edu.au (Giacinta Parish) 


\section{Introduction}

Ionophore-doped membranes are widely used for functionalisation in ion sensing technologies such as ion-selective electrodes (ISEs). The earliest work based on electrically neutral ionophores comes from the observation by Moore and Pressman that the antibiotic valinomycin is highly selective towards $\mathrm{K}^{+}$ ions [1]. This led to tremendous advances from the 1960s and onwards with the development of a range of sensors with natural or synthetic ionophores for a plethora of different types of ions $[2,3,4]$.

The desire to further miniaturise sensors, and improve their versatility and ruggedness for application outside the lab environment, has led to the development of solid-contact sensors such as coated-wire electrodes (CWEs) and ion-selective field effect transistors (ISFETs). In the 1970s, the CWE was introduced in which an ion-selective membrane was directly contacted with a metal wire $[5,6]$. Concurrently, the ISFET concept was also introduced

in which a membrane was deposited on a Si-based transistor in place of the metal gate $[7,8]$. However, these sensors suffered from poor stability and potential drift, as well as poor membrane adhesion [9]. In the late 1980s and early 1990s, these issues were partially resolved by introducing conductive polymers and a hydrogel as inter-layers for CWEs and Si-based ISFETs respectively $[10,11]$.

Recently, a new type of ISFET has been introduced based on AlGaN/GaN transistors, which appears to be a promising alternative to Si-based transistors, having a higher device transconductance and improved chemical stability $[12,13]$. A reference electrode free configuration has also been demonstrated to work well for $\mathrm{AlGaN} / \mathrm{GaN}$ sensors, where changes in gate charge are sensed through measuring the changes in the conductivity of the electron channel $[14,15]$.

As early as 1937, the very first theoretical description of ion-selective membranes was given by Nikolskii for glass ISEs [16]. This led to the well-known semi-empirical equation: the Nikolskii-Eisenman (N-E) equation, which relates the concentration of the ion of interest to the equilibrium potential response. A critical feature of Nikolskii's earlier work and the later $\mathrm{N}-\mathrm{E}$ description is that it provided a formal definition of the selectivity of ISEs towards different ions. However, it is based on equilibrium assumptions and cannot provide insight into fundamental ion charging mechanisms [17].

Presently, the most generalised description of ISEs is the Nernst-PlanckPoisson (NPP) model which has been developed by various researchers $[18$, 
19, 20], based on the pioneering work of Brumleve and Buck [21]. This model contains the least number of assumptions and can be used to provide greater insight into the underlying ion charging mechanisms in the membrane. Recent developments led to the incorporation of reaction terms in the NPP equations [22] that allowed for the description of ion-ionophore complexation, which is vital for greater discrimination between ions. However, the application of the NPP model was mainly in the context of traditional ISEs containing an inner filling solution and a suspended membrane. There is earlier work in which the model was applied to a CWE [23], but it was applied to the specific context where a conductive polymer was used as an inter-layer [22] and ion-ionophore complexation was not considered [22].

This work aims to clarify the understanding of how the membrane itself affects the response of solid-contact sensors, using the NPP model. This is achieved by making the assumption that the solid-contact completely blocks ion transfer (i.e. blocked interface) and does not interact electrostatically. Ion-ionophore complexation rates are included in the model [22], providing an understanding of their role. The ion charging mechanisms in the membrane and the boundary layer behaviour exhibited at the membrane|solution interface are studied, and the fundamental sensing principles elucidated. Simulations were conducted to make comparisons between the response of a sensor with a perfectly blocked interface and a traditional ISE. For the first time, we use the NPP model to analyse experimental results for AlGaN/GaN ISFETs with ionophore-doped membranes, achieving good qualitative agreement and model validation.

\section{Theory and model}

\subsection{Context}

The primary focus of this paper is the solution|membrane|solid-contact system illustrated in Figure 1(a). This is in contrast to the ISE setup with inner filling solution and a suspended membrane investigated by Jasielec et al. [22], and depicted in Figure 1(b).

Ions transfer into the membrane phase through solvation processes, described by the heterogenous rates of phase transfer (see Figure 1). The mobile ionic sites, $\mathrm{R}^{-}$, provide for neutrality in the bulk membrane and thus suppress interference of counter ions, $\mathrm{X}^{-}$, from the solution [3]. The ionophore, $\mathrm{L}$, binds with the target ion, $\mathrm{M}^{+}$, forming the highly stable $\mathrm{ML}^{+}$complex. The interfering ion, $\mathrm{I}^{+}$, forms less stable complexes with the ionophore, $\mathrm{IL}^{+}$, 
allowing for the discrimination of ions. Diffusion generated flux components occur in the system because of the spatial variations in concentrations of various species over time. At equilibrium, there is effectively a balance between diffusion driven flux components and electrically driven flux components caused by the separation of ionic charge. These processes are the same for the setups in both Figure 1(a) and Figure 1(b). However, the key distinction is that for the setup in Figure 1(b) solvation processes also occur between the membrane and the inner filling solution, whereas this does not occur for the system in Figure 1(a).

\subsection{Suitable Scales}

The natural scales used for the NPP equations are the thickness of the membrane for scaling in space and the slow characteristic diffusion time for scaling in time:

$$
\begin{aligned}
x^{\prime} & =\frac{x}{L_{m}} \\
t^{\prime} & =\frac{t}{\tau_{D}}
\end{aligned}
$$

where $L_{m}$ is the thickness of the membrane and $\tau_{D}$ is the characteristic diffusion time. The characteristic diffusion time is defined as $\tau_{D}=L_{m}{ }^{2} / D_{0}$, where $D_{0}$ is the characteristic diffusion coefficient which is used to scale the diffusion coefficients for the different species, leading to $D_{i}^{\prime}=D_{i} / D_{0}$ for the $i^{\text {th }}$ species.

The concentrations are scaled by the maximum concentration in the system, $C_{\text {max }}$, leading to $C_{i}^{\prime}=C_{i} / C_{0}$ for the $i^{t h}$ species. By choosing the diffusion process as the time scale, the natural scale which arises for flux is the characteristic diffusion flux, $J_{D}=D_{0} C_{\max } / L_{m}$, leading to $J_{i}^{\prime}=J_{i} / J_{D}$. The natural scaling for the electrical potential is the thermal potential, $\psi_{\text {th }}=R T / F$, where $R$ is the ideal gas constant, $T$ the absolute temperature, and $F$ is Faraday's constant, which leads to the scaled electric field, $E^{\prime}=E L_{m} / \psi_{t h}$.

\subsection{NPP System}

The NPP model consists of the flux equation, the mass conservation relation and Poisson's equation [22, 24]. With the scaling described previously the scaled dimensionless equations are [21, 22]:

$$
J_{i}^{\prime}\left(x^{\prime}, t^{\prime}\right)=-D_{i}^{\prime}\left[\frac{\partial C_{i}^{\prime}\left(x^{\prime}, t^{\prime}\right)}{\partial x^{\prime}}-z_{i} C_{i}^{\prime}\left(x^{\prime}, t^{\prime}\right) E^{\prime}\left(x^{\prime}, t^{\prime}\right)\right]
$$




$$
\begin{gathered}
\frac{\partial C_{i}^{\prime}\left(x^{\prime}, t^{\prime}\right)}{\partial t^{\prime}}=-\frac{\partial J_{i}^{\prime}\left(x^{\prime}, t^{\prime}\right)}{\partial x^{\prime}}+\text { Reactions } \\
\kappa \frac{\partial E^{\prime}\left(x^{\prime}, t^{\prime}\right)}{\partial x^{\prime}}=\sum_{\forall i} z_{i} C_{i}^{\prime}\left(x^{\prime}, t^{\prime}\right)
\end{gathered}
$$

where $J_{i}^{\prime}$ is the scaled flux of the $i^{\text {th }}$ species, $C_{i}^{\prime}$ is the scaled concentration of the $i^{t h}$ species, $E^{\prime}$ is the scaled electric field, $D_{i}^{\prime}$ is the scaled diffusion coefficient of the $i^{\text {th }}$ species, $z_{i}$ is the charge number of the $i^{t h}$ species, and $\kappa$ is the ratio of the electrical relaxation time scale over the diffusion time scale [25]. The reaction terms correspond to the complexation processes [22]. In the following discussion we will continue to use the scaled terms and drop the primes, to reduce the burden of notation.

Similar to the work of Cohen and Cooley, who were the first to provide a numerical solution to the NPP problem [25], the displacement current equation is used instead of Poisson's equation (5) for mathematical convenience:

$$
\kappa \frac{\partial E(x, t)}{\partial t}=\left[I-\sum_{\forall i} z_{i} J_{i}(x, t)\right]
$$

The electrical potential, $\psi$, can be computed in the usual manner by:

$$
\psi=\int E d x
$$

\subsection{Boundary Conditions}

The Chang-Jaffé flux boundary condition is used for the membrane|solution interface (see Figure 1(a)) [21]:

$$
J_{i}(0, t)=k_{i, f} C_{i, L e f t}-k_{i, b} C_{i}(0, t)
$$

where $k_{i, f}$ and $k_{i, b}$ are the scaled heterogenous rate constants for the $i^{\text {th }}$ species, $C_{i, \text { Left }}$ is the concentration of the $i^{\text {th }}$ species in the external solution and $C_{i}(0, t)$ is the concentration of the $i^{t h}$ species in the membrane at the membrane|solution interface (see Figure 1).

The ratio of the forward and backward heterogeneous rates is the partition constant of ionic transfer. For example, for the ion $\mathrm{M}^{+}$this transfer can be represented by:

$$
\mathrm{M}_{(\mathrm{aq})}^{+} \rightleftharpoons \mathrm{M}_{(\mathrm{mem})}^{+}
$$


where the partition constant, $k_{\mathrm{M}^{+}}$, is:

$$
k_{\mathrm{M}^{+}}=\frac{\left[\mathrm{M}_{(\mathrm{mem})}^{+}\right]}{\left[\mathrm{M}_{(\mathrm{aq})}^{+}\right]}=\frac{k_{\mathrm{M}^{+}, f}}{k_{\mathrm{M}^{+}, b}}
$$

The null flux condition is used at the membrane|solid-contact interface (see Figure 1(a)). This condition assumes that the solid-contact completely blocks mass transport of species and does not interact electrostatically. That is, we do not consider the boundary layers formed due to the specific solidcontact material [9]. This allows a focus on how the membrane itself affects the response of solid-contact sensors.

\subsection{Implications of the NPP model}

The dimensionless parameter $\kappa$ in (5) and (6) is critical because it is the ratio of the extremely fast electrical relaxation time scale to the extremely slow diffusion time scale. This parameter is defined here as [21, 25]:

$$
\kappa=\frac{\epsilon R T}{L_{m}^{2} F^{2} C_{\max }}
$$

where $\epsilon$ is the absolute permittivity of the medium.

At room temperature, diffusion is a significantly slower process than electrical effects and thus $\kappa$ is extremely small. For example, for a $15 \mu \mathrm{m}$ membrane with absolute permittivity $\epsilon=2.11 \times 10^{-11} \mathrm{Fm}^{-1}$ in a $0.1 \mathrm{M}$ buffer solution, $\kappa$ is approximately $10^{-8}$. Since the small $\kappa$ value is the multiplier for a derivative term in (5) the membrane exhibits boundary layer behaviour $[25,26]$, with the term on the left hand side of (5) vanishing for the bulk membrane giving $[25,26]$ :

$$
0=\sum_{\forall i} z_{i} C_{i}(x, t)
$$

That is, in the bulk of the membrane electro-neutrality holds. Electroneutrality in the bulk membrane does not arise by any a priori assumption. Rather, it is a direct consequence of the extremely different time scales involved. Electro-neutrality does not hold locally near the membrane|solution interface, where the interfacial kinetics (i.e. solvation processes) can generate a large local electric field gradient and the derivative term in (5) is no longer negligible $[25,26]$. 
The characteristic Debye length, $\lambda$, is directly related to $\kappa$ and is determined by:

$$
\lambda=\sqrt{\kappa} L_{m}
$$

The characteristic Debye length is significantly small in comparison to the membrane thickness and is an indication of the length scale of the boundary layer formed locally at the membrane|solution interface. As a comparison, a typical membrane thickness is $15 \mu \mathrm{m}$ and a typical Debye length is $1 \mathrm{~nm}$. Therefore, a significant charge and potential distribution arises within a few nanometres or a few tens of nanometres from the membrane|solution interface. ${ }^{1}$

\subsection{Reactions}

Reaction terms are incorporated in the mass conservation relation (4) following the approach by Jasielec et al. in [22]. The complexation of the ion, $\mathrm{M}^{+}$, with the neutral ionophore, $\mathrm{L}$, can be described by the following chemical reaction (see Figure 1):

$$
\mathrm{M}^{+}+\mathrm{L} \rightleftharpoons \mathrm{ML}^{+}
$$

where the equilibrium constant, $\beta_{\mathrm{ML}^{+}}$, is related to the association rate, $K_{\mathrm{M}^{+}, A}$, and disassociation rate, $K_{\mathrm{ML}^{+}, D}$, of complexation as follows:

$$
\beta_{\mathrm{ML}^{+}}=\frac{\left[\mathrm{ML}^{+}\right]}{\left[\mathrm{M}^{+}\right][\mathrm{L}]}=\frac{K_{\mathrm{M}^{+}, A}}{K_{\mathrm{ML}^{+}, D}}
$$

Thus, incorporation of complexation into the mass conservation relation will appear as [22]:

$$
\begin{aligned}
\frac{\partial C_{\mathrm{M}^{+}}}{\partial t} & =-\frac{\partial J_{\mathrm{M}^{+}}}{\partial x}+K_{\mathrm{ML}^{+}, D} C_{\mathrm{ML}^{+}}-K_{\mathrm{M}^{+}, A} C_{\mathrm{M}^{+}} C_{\mathrm{L}} \\
\frac{\partial C_{\mathrm{ML}^{+}}}{\partial t} & =-\frac{\partial J_{\mathrm{ML}^{+}}}{\partial x}-K_{\mathrm{ML}^{+}, D} C_{\mathrm{ML}^{+}}+K_{\mathrm{M}^{+}, A} C_{\mathrm{M}^{+}} C_{\mathrm{L}} \\
\frac{\partial C_{\mathrm{L}}}{\partial t} & =-\frac{\partial J_{\mathrm{L}}}{\partial x}+\sum_{\forall i o n s}\left(K_{i L, D} C_{i L}-K_{i, A} C_{i} C_{L}\right)
\end{aligned}
$$

\footnotetext{
${ }^{1}$ Refer to simulated spatial profiles in Figure 4 in Section 3.4 for an indication of the length scale of the boundary layer at the membrane|solution interface.
} 
The incorporation of complexation rates introduces an intermediary time scale in the model, which is intermediate in magnitude between the diffusive and electrical time scales. At equilibrium there will be a balance not only between the interfacial kinetics (i.e. solvation processes), and the diffusive and electrically driven fluxes, but also with the association and dissociation terms in equations (14) - (16).

\subsection{Model Implementation}

The model is implemented in MATLAB using the numerical method of lines (MOL) based on published work [18, 19, 24]. The discretisation scheme and algorithm used is identical to the MATLAB implementation by Grysakowski et al. in [18], but additionally incorporates information on ionionophore complexation following the work of Jasielec et al. [22, 27]. The model utilises MATLAB's inbuilt stiff integrator, ode15s, for solving the resulting system of spatially discretised ODEs in time.

\section{Results and Discussion}

\subsection{Comparison between ISE (with inner filling solution) and a membrane with a blocked interface}

Table 1: Parameters used for simulations

\begin{tabular}{lcccccccc}
\hline & & & & & & \\
$i$ & $C_{i, \text { Left }}(\mathrm{M})$ & $C_{i, \text { Right }}(\mathrm{M})$ (for ISE only) & $C_{i, 0}(\mathrm{~mol} / \mathrm{L})$ & $D_{i}\left(\mathrm{~m}^{2} \mathrm{~s}^{-1}\right)$ & $k_{i, f}\left(\mathrm{~ms}^{-1}\right)$ & $k_{i, b}\left(\mathrm{~ms}^{-1}\right)$ & $K_{i, A}\left(\mathrm{~m}^{3} \mathrm{~mol}^{-1} \mathrm{~s}^{-1}\right)$ & $K_{i, D}\left(\mathrm{~s}^{-1}\right)$ \\
\hline & & & & & & & \\
$\mathrm{M}^{+}$ & {$[\mathrm{a}]$} & {$[\mathrm{b}]$} & 0 & $10^{-11}$ & $10^{-3}$ & 1 & $10^{8}$ & \\
$\mathrm{I}^{+}$ & 0 & 0.1 & 0 & $10^{-11}$ & $10^{-4}$ & 1 & $10^{4}$ & \\
$\mathrm{R}^{-}$ & 0 & 0 & $10^{-3}$ & $10^{-11}$ & 0 & 0 & & $10^{4}$ \\
$\mathrm{~L}$ & 0 & 0 & $10^{-3}$ & $10^{-12}$ & 0 & 0 & & $10^{4}$ \\
$\mathrm{ML}^{+}$ & 0 & 0 & 0 & $10^{-12}$ & 0 & 0 & & 0 \\
$\mathrm{IL}^{+}$ & 0 & 0 & 0 & 12 & 0 & & \\
\hline
\end{tabular}

[a] Varied between $10^{-8}-10^{-2} \mathrm{M}$ for response curves.

[b] For ISE with inner solution, fixed at $0.01 \mathrm{M}$ for classical ISE response and $10^{-10} \mathrm{M}$ for super-Nernstian response, and not applicable for blocked interface sensor.

This subsection addresses the differences between ISEs with an inner filling solution (see Figure 1(b)) and solid-contact sensors with a blocked interface, which are the primary focus of this work (see Figure 1(a)). For the simulations in this subsection no interfering ions, $\mathrm{I}^{+}$, are present in the external solution. However, $0.1 \mathrm{M} \mathrm{I}^{+}$is present in the inner filling solution of the ISE, as in other studies [20, 22, 28, 29]. Throughout this paper a $15 \mu \mathrm{m}$ thick membrane with absolute permittivity $\epsilon=2.11 \times 10^{-10} \mathrm{Fm}^{-1}$ is assumed 
$[20,22]$. The concentration values of the various species in the membrane are initialised to ensure electro-neutrality, which is analogous to the preconditioning process in experiments. The parameters and initial values for the simulations in this subsection are as given in Table 1. Note that initially the total molar concentration of ionophore, L, in the membrane (complexed and uncomplexed forms, see Table 1) is double that of the anionic sites. This is approximately the optimal molar composition to ensure selectivity and Donnan exclusion, given that a monovalent target ion, $\mathrm{M}^{+}$, and a one-toone stoichiometry of ion-ionophore complexes are used in these test studies $[2,30]$.

For both types of sensors, the simulation results of the potential response as a function of the concentration of the target ion, $\mathrm{M}^{+}$, in the external solution are given in Figure 2. The response curves were shifted vertically, so a meaningful comparison can be made between the shape of the curves for the different situations depicted (see Figure 2). It can be seen that the lower detection limit of the classical ISE, with $0.01 \mathrm{M} \mathrm{M}^{+}$in the inner filling solution, is marginally better (lower) than that of the sensor with a blocked interface (see Figure 2(a)). This result agrees with documented findings on the similarity of the detection ranges between blocked interface sensors and classical ISEs [6, 9, 31].

It is also seen from Figure 2 that the super-Nernstian ISE, with a significantly lower concentration in the inner filling solution, here $10^{-10} \mathrm{M} \mathrm{M}^{+}$, has a worse detection limit with the linear Nernstian range beginning at concentrations of approximately $10^{-5} \mathrm{M} \mathrm{M}^{+}$in the external solution. This is in agreement with other studies in the literature on the super-Nernstian response [28, 29]. Further, the super-Nernstian response takes an extremely long time to equilibrate (see Figure 2(b)). In fact, the super-Nernstian response requires a measuring time of approximately $300 \mathrm{~s}$ to reach equilibrium [20]. Thus, the detection limits and sensor response for the ISEs are highly dependant on the concentration of ions in the inner filling solution. This is due to the additional solvation processes occurring at the membrane|inner solution boundary (see Figure 1(b)), and the transmembrane fluxes generated due to the presence of the inner filling solution [28, 29].

\subsection{Influence of complexation on the lower detection limit}

The effects of ion-ionophore complexation on the lower detection limits of membranes with a blocked interface are investigated. Different synthetic or natural ionophores can form complexes with ions at varying degrees of 
stability [3, 4]. The parameters and initial values used for the simulations in this subsection are the same as that in Table 1 for the blocked interface sensor, except that here $0.1 \mathrm{M}$ concentration of the interfering ion, $\mathrm{I}^{+}$, is present in the external solution. The association rates of complexation are varied for both the target and interfering ions, in order to vary the stability constants, as shown in Figure 3.

In Figure 3(a), the model is validated by the well-known finding that the greater the stability of the target ion complex, the better (lower) the detection limit $[3,4]$. It is observed that by increasing the stability of the target ion complex, $\mathrm{ML}^{+}$, by an order of magnitude the lower detection limit decreases by an order of magnitude, given the parameter values used for these simulations (see Figure 3(a)). Model predictions are further validated in Figure 3(b), by the well-known finding that greater stability for the interfering ion complex, $\mathrm{IL}^{+}$, results in a worse (increased) lower detection limit. These simulations are in alignment with observations by Jasielec et al., for ISEs with an inner filling solution [22].

\subsection{Ion charging mechanisms}

The ion charging mechanisms of the membrane and the boundary layer behaviour are studied here in depth. These mechanisms are revealed by conducting simulations and depicting the spatial distribution of species in the membrane and the electrical properties at equilibrium (see Figure 4) and as a function of time (see Figure 5). The simulations for this subsection again use the same parameters and initial conditions in Table 1 for blocked interface sensors, except that here $0.1 \mathrm{M}$ concentration of the interfering ion, $\mathrm{I}^{+}$, is present in the external solution.

Due to the high stability of the target ion complex, $\beta_{\mathrm{ML}^{+}}=10^{4}$, the target ion is predominantly in $\mathrm{ML}^{+}$form in the membrane. This can be seen in Figure 4(a) and Figure 4(b) where the steady state spatial profiles of the concentration of the target ion, $\mathrm{M}^{+}$, and the target ion complex, $\mathrm{ML}^{+}$are depicted, noting the different scales used for the vertical axis.

When the sensor is first immersed in the external solution, the interfacial heterogenous rates drive $\mathrm{M}^{+}$to exit the membrane phase (see Figure 4(a)), leaving behind the mobile anionic sites (see Figure $4(\mathrm{~b})$ ), $\mathrm{R}^{-}$, due to the absence of $\mathrm{M}^{+}$in the external solution. This in turn will drive the disassociation of the $\mathrm{ML}^{+}$complex near the membrane|solution interface at $x=0$, to maintain the equilibrium constant of complexation (see Figure 4(a)-(b)). 
The reduced $\mathrm{M}^{+}$and $\mathrm{ML}^{+}$at the interface will generate a diffusion flux component directed from the bulk membrane, which at equilibrium will balance the opposing electrically driven flux component, giving zero net flux for each species (see Figure 4(a)-(b)). As the concentration of $\mathrm{M}^{+}$in the external solution increases, the hetergenous rates drive $\mathrm{M}^{+}$into the membrane and $\mathrm{ML}^{+}$complexes are formed. Both species diffuse a short distance in the membrane prior to being balanced by the electrical effects (see Figure 4(a)(b)). Due to the different time scales between the electrical and diffusive processes, a thin boundary layer (i.e. space-charge region) is formed at the membrane|solution interface at $x=0$ (see Figure 4(a)-(c)). The entire charge and potential distribution arises within approximately the first $15 \mathrm{~nm}$ (see Figure 4(e)-(f)).

These processes are clearly illustrated by the transient simulations in Figure 5(a) and Figure 5(b), depicting the non-equilibrium situation at the instant the concentration of $\mathrm{M}^{+}$in the external solution is stepped from $10^{-4} \mathrm{M}$ to $10^{-3} \mathrm{M}$. Here the increases in $\mathrm{ML}^{+}$and the electrical potential in the boundary layer are depicted over time. Equilibrium is reached very rapidly in this model, as seen in Figure 5. It may be expected that in practical situations reaching equilibrium will take longer since diffusion layers also form in the solution [24]. Also, there may be boundary layers formed due to the electrostatic interaction of the particular solid-contact [9]. However, these factors are not taken into account in order to focus on the basic principles of ion-selection of the membrane and its influence on solid-contact sensors.

In contrast to previous phase boundary [32], and diffuse double layer $[33,34]$ models for ionophore-doped membranes, the NPP model here does not make any equilibrium assumptions. In this model, the equilibrium profiles in Figure 4 are obtained by solving temporally over a long time period. Also, we do not make any initial assumption of the existence of a double layer, rather it arises naturally as a consequence of the diffusion and electrical processes. In fact, the depicted boundary layer is typical of singular mathematical systems where an extremely small or large coefficient is a multiplier of the highest derivative term [35], for instance in Equation (5). The length of the space charge region, for the simulations in Figure 4 and Figure 5, is comparable to the profiles obtained in previous NPP treatments $[20,21,36]$. However, those treatments did not take into account complexation with neutral carriers. This work builds on the model by Jaiselec et al. for ISEs [22], which incorporated complexation rates, but here we focus on blocked interface sensors and study the boundary layer in greater 
depth across different species. The model description provided in this work is useful, as it provides clarity into the dynamic interplay between the fundamental interfacial kinetic, diffusion, electrical, and complexation processes on the sensor response.

\subsection{Comparison with experimental results}

Following from the previously discussed simulation studies, experimental data was obtained for validation of the model. For this work, experimental results were obtained for $\mathrm{AlGaN} / \mathrm{GaN}$ ISFETs functionalised by ionophoredoped membranes, selective to the divalent $\mathrm{Ca}^{2+}$ target ion. This is in contrast to the previous simulation studies of this work where a monovalent target ion was used. With the divalent target ion, the membrane must be initialised so the concentration of the analyte is half of the concentration of the anionic sites, to ensure everything is initially electro-neutral in the membrane. Again, the model makes the assumption that all ion-ionophore complexes form only 1:1 stoichiometries following [22].

A $15 \mu \mathrm{m}$ thick PVC membrane, containing Calcium Ionophore IV (ETH 5234) and tetrakis(4-chlorophenyl)borate $\left(\mathrm{T}_{4} \mathrm{ClPB}\right)$ anionic sites was used for the experiments. Approximately $44 \mathrm{~mol} \%$ of anionic sites relative to ionophore is used, which is similar to the composition used for the calcium experiments in [37].

As discussed earlier, AlGaN/GaN ISFETs have previously been demonstrated to successfully operate in a reference electrode free configuration for nitrate and mercury(II) ions [14, 15]. Figure 6(a) schematically indicates the layout of the devices. A constant current of $100 \mu \mathrm{A}$ was applied through the drain-source input. The zero gate bias electron density of the channel of the devices, as obtained from standard Hall measurements, is $n_{s}=8.257 \times 10^{12} \mathrm{~cm}^{-2}$ and the mobility is $\mu=1472 \mathrm{~cm}^{2} \mathrm{~V}^{-1} \mathrm{~s}^{-1}$.

In this configuration, changes in gate potential are determined by the changes in the channel conductance: $G_{\text {sense }}=1 / R_{\text {sense }}$ (see Figure $6(\mathrm{c})$ ). The total measured resistance, $R_{\text {total }}$, must take into account the parasitic resistances associated with the shunt and access regions (see Figure 6(b)). The relationship between $G_{\text {sense }}, R_{\text {total }}$ and the parasitic resistances can be derived as:

$$
G_{\text {sense }}=\frac{1}{R_{\text {total }}-R_{\text {access }}}-\frac{1}{R_{\text {shunt }}}
$$


where $R_{\text {access }}=305 \Omega$, and $R_{\text {shunt }}=7.7 \mathrm{k} \Omega$, given the dimensions of the layout (see Figure 6(a)) and previously described electron transport properties.

The relationship between the channel conductance and the gate potential for very small drain-source potential is [14]:

$$
G_{\text {sense }}=\frac{\left(V_{G}-V_{T}\right) \mu C W}{L}
$$

where $V_{G}$ is the gate potential, $V_{T}$ is the threshold voltage of the device, $C$ is the capacitance of the dielectric layer, $W$ is the width of the sensing region (see Figure 6), and $L$ is the length of the sensing region (see Figure 6).

The total capacitance per unit area of the dielectric layer is calculated to be $C=4.67 \mathrm{mFm}^{-2}$, given that the device under investigation had a mole fraction of $x=0.253$ for the AlGaN layer and thickness of $d_{A l G a N}=15.5 \mathrm{~nm}$ and a thickness of $d_{G a N}=2 \mathrm{~nm}$ for the GaN cap [14]. The relationship between the gate potential and the channel conductance, can now be deduced from Equation 18 as:

$$
V_{G}-V_{T}(m V)=\frac{G_{\text {sense }}(\mu S)}{0.687}
$$

Prior to measurements, the ISFET was preconditioned in $0.1 \mathrm{M} \mathrm{CaCl}_{2}$. During measurements the concentration of $\mathrm{CaCl}_{2}$ in the solution was varied from $10^{-8}-10^{-2} \mathrm{M}$ in $0.1 \mathrm{M} \mathrm{KCl}, \mathrm{NaCl}$, or $\mathrm{MgCl}_{2}$ ionic strength buffer solutions, which present $\mathrm{K}^{+}, \mathrm{Na}^{+}$and $\mathrm{Mg}^{2+}$ as potential interfering ions.

Table 2 gives the relevant parameters for the simulations of the experimental results. It is assumed that the absolute membrane permittivity is $\epsilon=2.11 \times 10^{-10} \mathrm{Fm}^{-1}[22,24]$. Following [22], due to the absence of experimentally determined values, the diffusion coefficients of free ions in the membrane were assumed to be two orders of magnitude lower than their corresponding diffusion coefficients in solution [38]. These values are approximately in the correct order of magnitude [39]. The diffusion coefficients for anionic sites in the membrane, the ionophore and the respective complexes with the target and interfering ion are assumed to be $10^{-12} \mathrm{~m}^{2} \mathrm{~s}^{-1}$ following [22], which is also approximately in the correct order of magnitude [40]. The heterogenous and complexation rates were estimated to fit the relevant experimental response curves.

Since for these experiments no reference electrode is used in the solution, the changes in the gate potential of the ISFET are determined indirectly using (19). Therefore, considering that each measured potential value is relative, 
Table 2: Parameters used for simulations to compare with experiment

\begin{tabular}{lccccccc}
\hline$i$ & $C_{i, \text { Left }}(\mathrm{M})$ & $C_{i, 0}(\mathrm{M})$ & $D_{i}\left(\mathrm{~m}^{2} \mathrm{~s}^{-1}\right)[\mathbf{a}]$ & $k_{i, f}\left(\mathrm{~ms}^{-1}\right)$ & $k_{i, b}\left(\mathrm{~ms}^{-1}\right)$ & $K_{i, A}\left(\mathrm{~m}^{3} \mathrm{~mol}^{-1} \mathrm{~s}^{-1}\right)$ & $K_{i, D}\left(\mathrm{~s}^{-1}\right)$ \\
\hline $\mathrm{Ca}^{2+}$ & {$[\mathbf{b}]$} & 0 & $0.792 \times 10^{-11}$ & $10^{-3}$ & 1 & $5 \times 10^{7}$ & \\
$\mathrm{Cl}^{-}$ & {$[\mathbf{c}]$} & 0 & $2.032 \times 10^{-11}$ & $2 \times 10^{-3}$ & $1.3 \times 10^{-1}$ & & \\
$\mathrm{R}^{-}$ & 0 & $6.5 \times 10^{-3}$ & $10^{-12}$ & 0 & 0 & & \\
$\mathrm{~L}$ & 0 & $11.15 \times 10^{-3}$ & $10^{-12}$ & 0 & 0 & & $10^{4}$ \\
$\mathrm{CaL}^{2+}$ & 0 & $3.25 \times 10^{-3}$ & $10^{-12}$ & 0 & 0 & $10^{2}$ & \\
$\mathrm{~K}^{+}$ & 0.1 & 0 & $1.957 \times 10^{-11}$ & $2.5 \times 10^{-4}$ & 1 & $10^{4}$ & \\
$\mathrm{Mg}^{2+}$ & 0.1 & 0 & $0.706 \times 10^{-11}$ & $2.5 \times 10^{-5}$ & 1 & $10^{3}$ & $10^{4}$ \\
$\mathrm{Na}^{+}$ & 0.1 & 0 & $1.334 \times 10^{-11}$ & $9 \times 10^{-5}$ & 1 & & $10^{4}$ \\
$\mathrm{KL}^{+}$ & 0 & 0 & $10^{-12}$ & 0 & 0 & & $10^{4}$ \\
$\mathrm{MgL}^{2+}$ & 0 & 0 & $10^{-12}$ & 0 & 0 & & \\
$\mathrm{NaL}^{+}$ & 0 & 0 & $10^{-12}$ & 0 & 0 & & \\
\hline
\end{tabular}

[a] Following [22], the diffusion coefficients of anionic sites ionophore, and complexes in the membrane were assumed to be $10^{-12} \mathrm{~m}^{2} \mathrm{~s}^{-1}$, and the diffusion coefficients of free ions in the membrane were assumed to be two orders of magnitude lower than in solution [22]. [b] Varied between $10^{-8}-10^{-2} \mathrm{M}$ for response curves.

[c] Concentration varied such that electro-neutrality in the solution holds.

rather than the absolute value with respect to a datum, all measured and simulated response curves were shifted vertically for the purpose of making comparisons of the shapes between the response curves.

Figure 7 gives both the experimental value and the corresponding simulated values for the three ionic strength buffer solutions. The comparison between the experimental and simulation results in Figure 7 indicates a noticeably higher level of interference in the $\mathrm{MgCl}_{2}$ solution compared to the others. The higher interference level is expected, as calcium ionophore IV forms a comparatively more stable complex with the interfering $\mathrm{Mg}^{2+}$ ion, in comparison to the $\mathrm{K}^{+}$and $\mathrm{Na}^{+}$ions [41]. The equilibrium constants, determined from the estimated complexation rates (see Table 1), are $\beta_{\mathrm{KL}^{+}}=10^{-2}$, $\beta_{\mathrm{NaL}^{+}}=10^{-1}$ and $\beta_{\mathrm{MgL}^{2+}}=1$. These estimations reflect the degree of interference the ion poses due to its ability to form complexes. Determination of a precise value for these equilibrium constants may be obtained later through impedance spectroscopy measurements and solving the inverse problem with the model $[42,43]$.

The response curves exhibit a near Nernstian response, like in previous studies for $\mathrm{AlGaN} / \mathrm{GaN}$ sensors $[15,14]$, in this case for divalent target ions. The slopes are 23, 24 and $19 \mathrm{mV}$ in $\mathrm{KCl}, \mathrm{NaCl}$ and $\mathrm{MgCl}_{2}$ solutions respectively. In many cases cited in the literature a formal Nernstian slope is not exhibited due to the contamination of other ions [33]. In this case the slightly 
lower slopes is likely due to a noticeable uptake of the $\mathrm{Cl}^{-}$counter ion. This is predicted by the faster forward heterogeneous rate of $2 \times 10^{-3} \mathrm{~ms}^{-1}$ and the slower backward rate of $0.13 \mathrm{~ms}^{-1}$ to fit the data, when compared to the forward rate of $10^{-4} \mathrm{~ms}^{-1}$ and backward rate of $1 \mathrm{~ms}^{-1}$ used by Jasielec et al. $[22]$.

The significant deviations near the upper detection limits, particularly for the $\mathrm{KCl}$ solution, suggest that the model is limited for the description of the sensor behaviour at lower analyte concentrations. The upper detection limit is believed to be due to the onset of Donnan failure, where there is a loss of permselectivity of the membrane and there is bulk extraction of counter ions [3]. The limitation of the model to lower analyte concentrations may be circumvented by incorporating thermodynamic activity coefficients. The activity coefficients are particularly important at higher concentration values where the system will deviate from unity for ideal mixtures. Despite these differences, we achieve fairly good qualititative agreement between the model and the experimental data up to concentrations of $10^{-3} \mathrm{M}$ of the target ion in the solution.

\section{Conclusion}

Using the NPP model, we have successfully elucidated the influence of ionophore-doped membranes on the response of solid-contact sensors, by assuming an ideal blocked interface. For the first time, a comparison is made between blocked interface sensors and traditional ISEs using the NPP model, which is in alignment with experimental findings from the literature. The role of ion-ionophore complexation in influencing the lower detection limit is in alignment with previous work by Jasielec et al. for traditional ISEs [22]. The interplay between the fundamental diffusion, electrical, interfacial kinetics and the complexation processes responsible for the sensor behaviour is described with greater clarity with the NPP model.

Also for the first time, the NPP model is successfully applied to describe and analyse experimental results obtained for AlGaN/GaN ISFETs. The model qualitatively accounted for the experimental differences in the equilibrium responses of the sensor, in the presence of different interfering ions.

\section{Acknowledgement}

The experimental work was partially funded by the Australian Research Council, grant number DP140100827, and performed at the Western Aus- 
tralian Node of the Australian National Fabrication Facility, a company established under the National Collaborative Research Infrastructure Strategy to provide nano- and micro-fabrication facilities for Australia's researchers. The authors also gratefully acknowledge the help and support provided over email by Bartosz Grysakowski and Marek Danielewski from AGH University of Science and Technology in Poland, in assisting with troubleshooting during code development for the simulations.

\section{References}

[1] C. Moore, B. C. Pressman, Mechanism of action of valinomycin on mitochondria, Biochemical and Biophysical Research Communications 15 (1964) $562-567$.

[2] E. Bakker, P. Bühlmann, E. Pretsch, Carrier-Based Ion-Selective Electrodes and Bulk Optodes: Part 1. General Characteristics, Chemical Reviews 97 (1997) 3083 - 3132.

[3] P. Bühlmann, E. Pretsch, E. Bakker, Carrier-Based Ion-Selective Electrodes and Bulk Optodes: Part 2. Ionophores for Potentiometric and Optical Sensors, Chemical Reviews 98 (1998) 1593 - 1688.

[4] W. E. Morf, CHAPTER 1 - Introduction and Preliminary Aspects, in: The Principles of Ion-Selective Electrodes and of Membrane Transport, volume 2 of Studies in Analytical Chemistry, Elsevier, 1981, pp. $1-24$.

[5] R. W. Cattrall, H. Freiser, Coated wire ion-selective electrodes, Analytical Chemistry 43 (1971) 1905-1906.

[6] H. J. James, G. Carmack, H. Freiser, Coated wire ion-selective electrodes, Analytical Chemistry 44 (1972) 856-857.

[7] P. Bergveld, Development of an Ion-Sensitive Solid-State Device for Neurophysiological Measurements, IEEE Transactions on Biomedical Engineering BME-17 (1970) 70 - 71.

[8] S. D. Moss, J. Janata, C. C. Johnson, Potassium ion-sensitive field effect transistor, Analytical Chemistry 47 (1975) 2238 - 2243. 
[9] J. Hu, A. Stein, P. Bühlmann, Rational design of all-solid-state ionselective electrodes and reference electrodes, TrAC Trends in Analytical Chemistry 76 (2016) $102-114$.

[10] J. Bobacka, A. Ivaska, A. Lewenstam, Potentiometric Ion Sensors Based on Conducting Polymers, Electroanalysis 15 (2003) 366-374.

[11] E. J. Sudhlter, P. D. van der Wal, M. Skowronska-Ptasinska, A. van den Berg, P. Bergveld, D. N. Reinhoudt, Modification of ISFETs by covalent anchoring of poly(hyroxyethyl methacrylate) hydrogel. Introduction of a thermodynamically defined semiconductor-sensing membrane interface, Analytica Chimica Acta 230 (1990) 59 - 65.

[12] G. Steinhoff, O. Purrucker, M. Tanaka, M. Stutzmann, M. Eickhoff, AlxGa1xN a new material system for biosensors, Advanced Functional Materials 13 (2003) 841-846.

[13] G. Steinhoff, B. Baur, G. Wrobel, S. Ingebrandt, A. Offenhusser, A. Dadgar, A. Krost, M. Stutzmann, M. Eickhoff, Recording of cell action potentials with $\mathrm{AlGaN} / \mathrm{GaN}$ field-effect transistors, Applied Physics Letters 86 (2005).

[14] M. Myers, F. L. M. Khir, A. Podolska, G. A. Umana-Membreno, B. Nener, M. Baker, G. Parish, Nitrate ion detection using AlGaN/GaN heterostructure-based devices without a reference electrode, Sensors and Actuators B: Chemical 181 (2013) $301-305$.

[15] M. Asadnia, M. Myers, N. Akhavan, K. O’Donnell, G. A. UmanaMembreno, U. Mishra, B. Nener, M. Baker, G. Parish, Mercury(II) selective sensors based on AlGaN/GaN transistors, Analytica Chimica Acta 943 (2016) $1-7$.

[16] B. Nikolskii, Theory of the glass electrode I, J. Phys. Chem (USSR) 10 (1937) $495-503$.

[17] A. Lewenstam, Non-equilibrium potentiometry-the very essence, Journal of Solid State Electrochemistry 15 (2011) $15-22$.

[18] B. Grysakowski, B. Bożek, M. Danielewski, Electro-Diffusion in Multicomponent Ion-Selective Membranes; Numerical Solution of the Coupled 
Nernst-Planck-Poisson Equations, Defect and Diffusion Forum 273-276 (2008) $113-118$.

[19] W. Kucza, M. Danielewski, A. Lewenstam, EIS simulations for ionselective site-based membranes by a numerical solution of the coupled Nernst Planck Poisson equations, Electrochemistry Communications 8 (2006) $416-420$.

[20] J. J. Jasielec, T. Sokalski, R. Filipek, A. Lewenstam, Comparison of different approaches to the description of the detection limit of ionselective electrodes, Electrochimica Acta 55 (2010) 6836 - 6848.

[21] T. R. Brumleve, R. P. Buck, Numerical solution of the Nernst-Planck and Poisson equation system with applications to membrane electrochemistry and solid state physics, Journal of Electroanalytical Chemistry 90 (1978) $1-31$.

[22] J. J. Jasielec, T. Sokalski, R. Filipek, A. Lewenstam, Neutral-Carrier Ion-Selective Electrodes Assessed by the Nernst-Planck-Poisson Model, Analytical Chemistry 87 (2015) 8665 - 8672.

[23] J. J. Jasielec, G. Lisak, M. Wagner, T. Sokalski, A. Lewenstam, NernstPlanck-Poisson Model for the Description of Behaviour of Solid-Contact Ion-Selective Electrodes at Low Analyte Concentration, Electroanalysis 25 (2013) $133-140$.

[24] J. J. Jasielec, R. Filipek, K. Szyszkiewicz, J. Fausek, M. Danielewski, A. Lewenstam, Computer simulations of electrodiffusion problems based on Nernst-Planck and Poisson equations, Computational Materials Science 63 (2012) $75-90$.

[25] J. Cohen, J. W. Cooley, The numerical solution of the time-dependant Nernst-Planck equations, Biophysical Journal 5 (1965) 145 - 162.

[26] D. P. Chen, J. W. Jerome, R. S. Eisenberg, V. Barcilon, Qualitative Properties of Steady-State Poisson-Nernst-Planck Systems: Perturbation and Simulation Study, SIAM Journal on Applied Mathematics 57 (1997) $631-648$. 
[27] J. J. Jasielec, R. Filipek, K. Szyszkiewicz, T. Sokalski, A. Lewenstam, Continuous modeling of calcium transport through biological membranes, Journal of Materials Engineering and Performance (2016) 1 -6 .

[28] P. Lingenfelter, I. Bedlechowicz-Sliwakowska, T. Sokalski, M. MajZurawska, A. Lewenstam, Time-Dependent Phenomena in the Potential Response of Ion-selective Electrodes Treated by the Nernst Planck Poisson Model. 1. Intramembrane Processes and Selectivity, Analytical Chemistry 78 (2006) 6783-6791.

[29] T. Sokalski, W. Kucza, M. Danielewski, A. Lewenstam, TimeDependent Phenomena in the Potential Response of Ion-Selective Electrodes Treated by the Nernst Planck Poisson Model. Part 2: Transmembrane Processes and Detection Limit, Analytical Chemistry 81 (2009) 5016-5022.

[30] R. Eugster, P. M. Gehrig, W. E. Morf, U. E. Spichiger, W. Simon, Selectivity-modifying influence of anionic sites in neutral-carrier-based membrane electrodes, Analytical Chemistry 63 (1991) 2285-2289.

[31] N. R. Stradiotto, H. Yamanaka, M. V. B. Zanoni, Electrochemical sensors: a powerful tool in analytical chemistry, Journal of the Brazilian Chemical Society 14 (2003) $159-173$.

[32] E. Bakker, P. Bhlmann, E. Pretsch, The phase-boundary potential model, Talanta 63 (2004) 3 -20. Molecular Recognition and Chemical Sensors.

[33] K. Tohda, T. Higuchi, D. Dragoe, Y. Umezawa, Threshold ionic site concentrations required for nernstian potentiometric responses of neutral ionophore-incorporated ion-selective liquid membranes, Analytical Sciences 17 (2001) 833-839.

[34] K. Tohda, D. Dragoe, M. Shibata, Y. Umezawa, Studies on the matched potential method for determining the selectivity coefficients of ionselective electrodes based on neutral ionophores: Experimental and theoretical verification, Analytical Sciences 17 (2001) 733-743.

[35] E. Hinch, Perturbation Methods, Cambridge Texts in Applied Mathematics, Cambridge University Press, 1991. 
[36] W. E. Morf, E. Pretsch, N. F. De Rooij, Theoretical treatment and numerical simulation of potential and concentration profiles in extremely thin non-electroneutral membranes used for ion-selective electrodes, Journal of electroanalytical chemistry (Lausanne, Switzerland) 642 (2010) 45-56.

[37] S. Bodor, J. M. Zook, E. Lindner, K. Tóth, R. E. Gyurcsányi, Chronopotentiometric method for the assessment of ionophore diffusion coefficients in solvent polymeric membranes, Journal of Solid State Electrochemistry 13 (2009) 171-179.

[38] E. Samson, J. Marchand, K. A. Snyder, Calculation of ionic diffusion coefficients on the basis of migration test results, Materials and Structures 36 (2003) $156-165$.

[39] L. Y. Heng, K. Toth, E. A. Hall, Ion-transport and diffusion coefficients of non-plasticised methacrylic-acrylic ion-selective membranes, Talanta 63 (2004) $73-87$.

[40] S. Bodor, J. M. Zook, E. Lindner, K. Toth, R. E. Gyurcsanyi, Electrochemical methods for the determination of the diffusion coefficient of ionophores and ionophore-ion complexes in plasticized PVC membranes, Analyst 133 (2008) $635-642$.

[41] J. Zhu, Y. Qin, Y. Zhang, Magnesium-selective ion-channel mimetic sensor with a traditional calcium ionophore, Analytical Chemistry 82 (2010) $436-440$.

[42] J. J. Jasielec, B. Wierzba, B. Grysakowski, T. Sokalski, M. Danielewski, A. Lewenstam, Novel strategy for finding the optimal parameters of ion selective electrodes, ECS Transactions 33 (2011) 19 - 29.

[43] B. Grysakowski, J. J. Jasielec, B. Wierzba, T. Sokalski, A. Lewenstam, M. Danielewski, Electrochemical impedance spectroscopy (EIS) of ion sensors: Direct modeling and inverse problem solving using the NernstPlanck-Poisson (NPP) model and the HGS (FP) optimization strategy, Journal of electroanalytical chemistry 662 (2011) 143 - 149. 


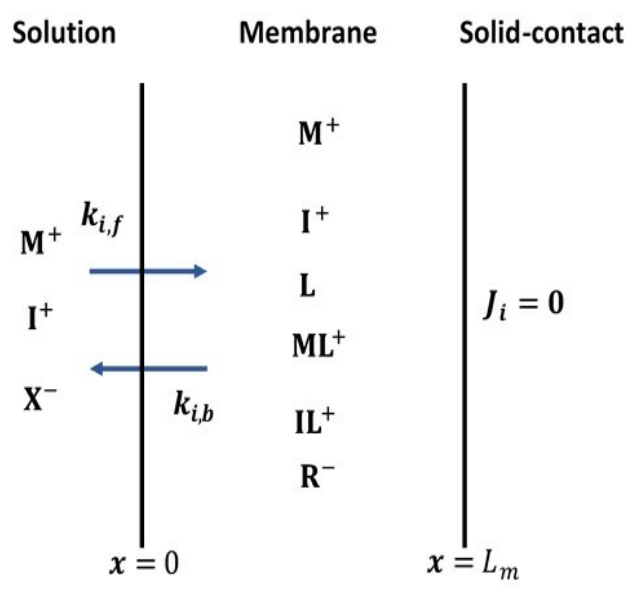

(a)
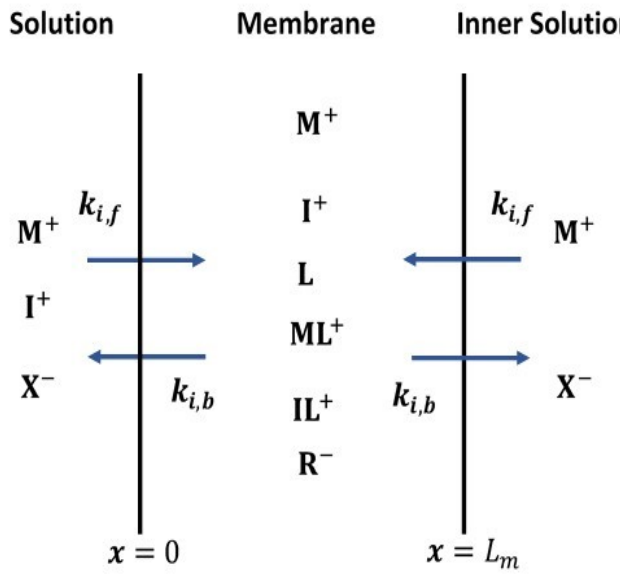

(b)

Figure 1: Comparison between (a) Solution|Membrane|Solid-contact system for this work and (b) Solution|Membrane|Inner solution system from [22], where $\mathrm{M}^{+}$is the target ion, $\mathrm{I}^{+}$ an interfering ion, $\mathrm{X}^{-}$a counter ion, $\mathrm{R}^{-}$the mobile anionic site, $\mathrm{L}$ the neutral ionophore, $\mathrm{ML}^{+}$the target ion complex, and $\mathrm{IL}^{+}$the interfering ion complex. The $k_{i, f}$ and $k_{i, b}$ parameters are the heterogenous rates of phase transfer for the $i^{t h}$ species and $L_{m}$ is the membrane thickness. 


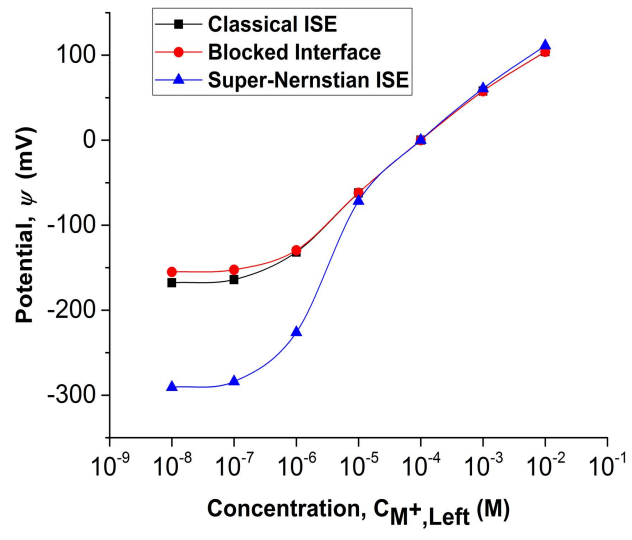

(a)

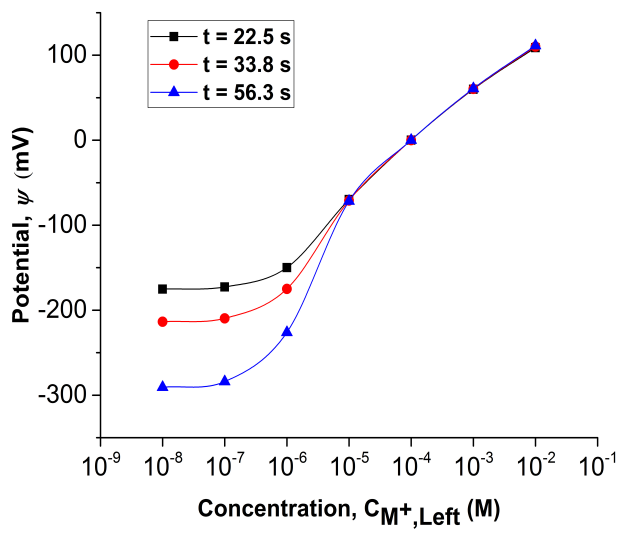

(b)

Figure 2: Response curves for (a) Classical ISE response with $0.01 \mathrm{M}$ of the target ion in the inner solution, super-Nernstian response with $10^{-10} \mathrm{M}$ of the target ion in the inner solution $[28,29]$ and an ideal blocked interface solid-contact sensor, and (b) the time dependence of the super-Nernstian ISE at $t=22.5,33.8$, and $56.25 \mathrm{~s}$ measured from the initial condition $[20,28,29] . C_{\mathrm{M}^{+}, \text {Left }}$ is the concentration of the target ion in the external solution.

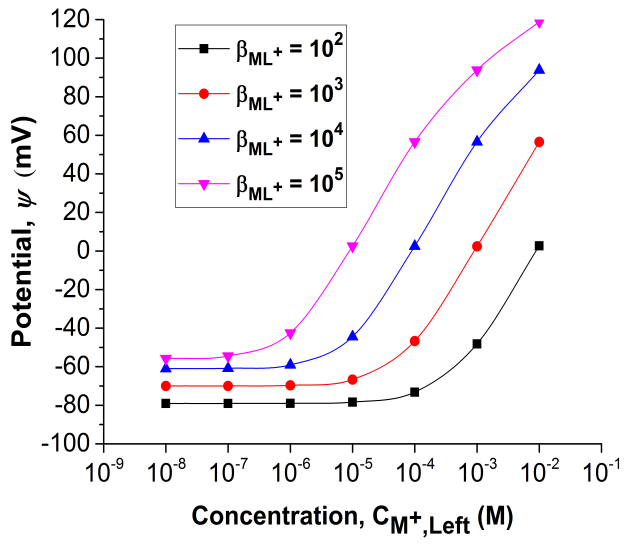

(a)

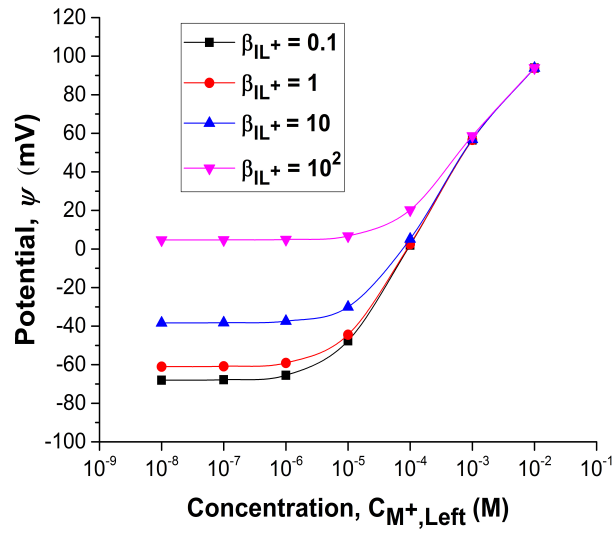

(b)

Figure 3: Effect of ion-ionophore complexation on lower detection limits by (a) varying equilibrium constant for the $\mathrm{ML}^{+}$complex between $\beta_{\mathrm{ML}^{+}}=10^{2}-10^{5}$ (b) varying equilibrium constant for the $\mathrm{IL}^{+}$complex between $\beta_{\mathrm{IL}^{+}}=10^{-1}-10^{2}$. 


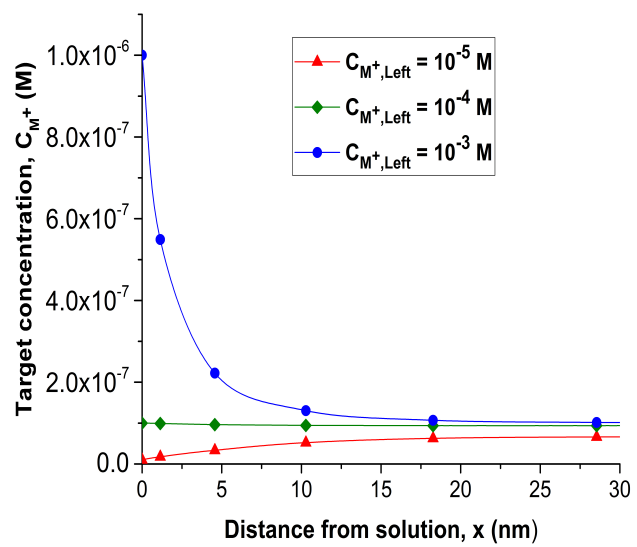

(a)

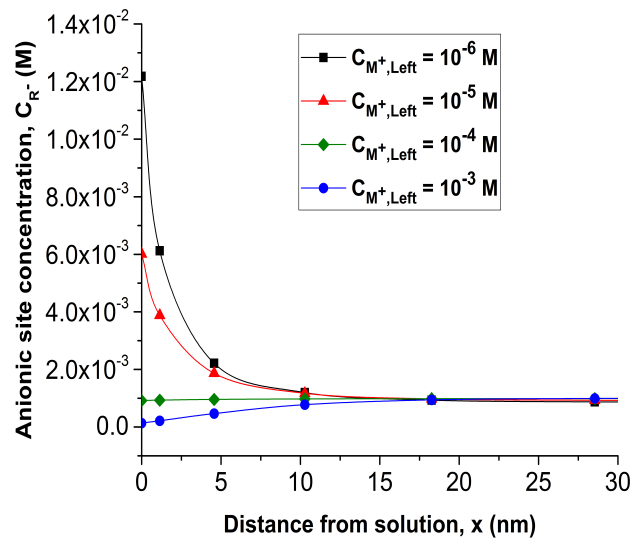

(c)

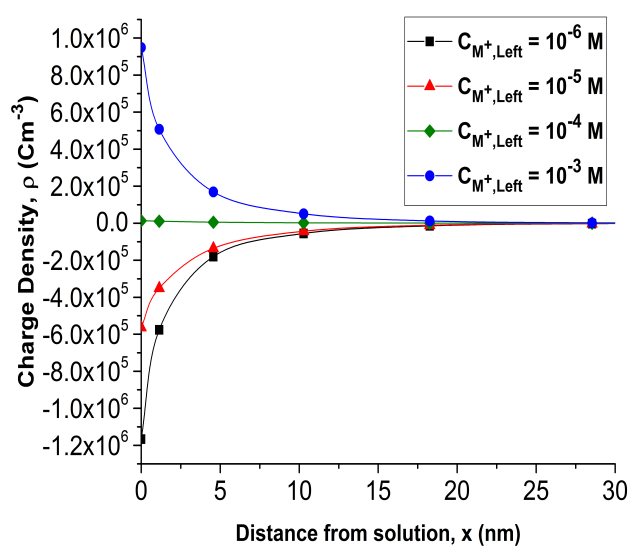

(e)

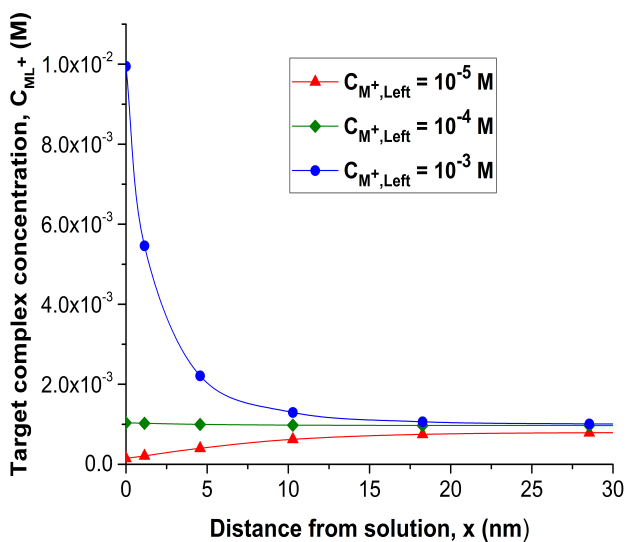

(b)

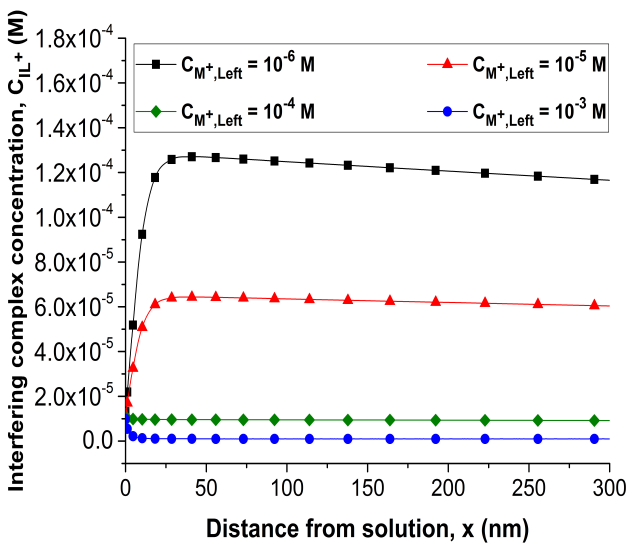

(d)

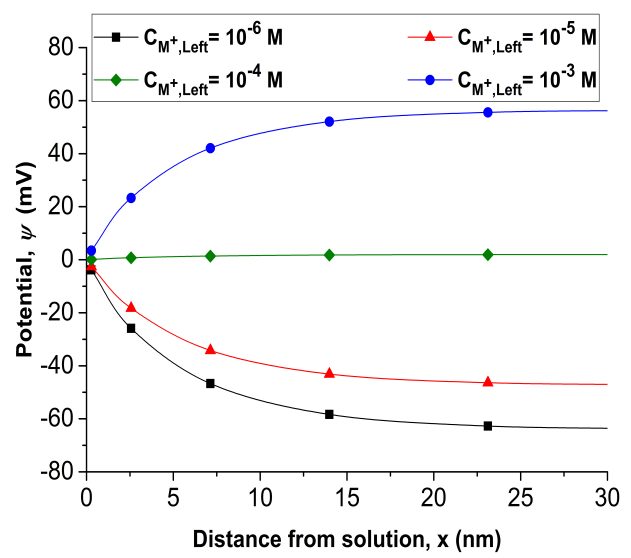

(f)

Figure 4: Equilibrium spatial profiles inside the membrane, moving away from the solution|membrane interface for (a) the target ion concentration, (b) the target ion complex concentration, (c) the anionic site concentration, (d) the interfering ion complex concentration, (e) the total charge density, and (f) the electrical potential. Note a different length scale is used for (d) to show that the interfering ion complex concentration does not necessarily level out like the other profiles in the bulk membrane. 


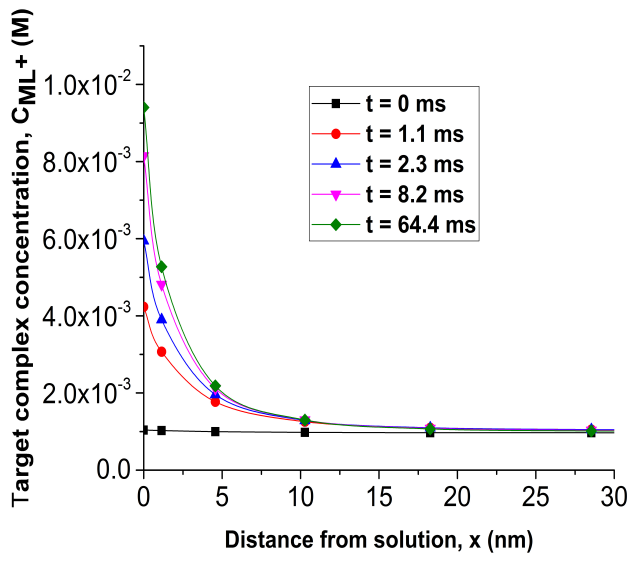

(a)

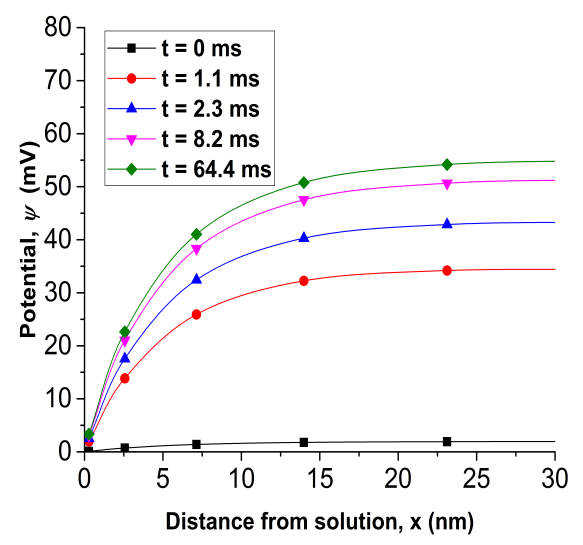

(b)

Figure 5: Simulated spatial profiles for (a) target ion complex concentration and (b) electrical potential at various times after the target ion concentration in the external solution is stepped from $10^{-4} \mathrm{M}$ to $10^{-3} \mathrm{M}$.

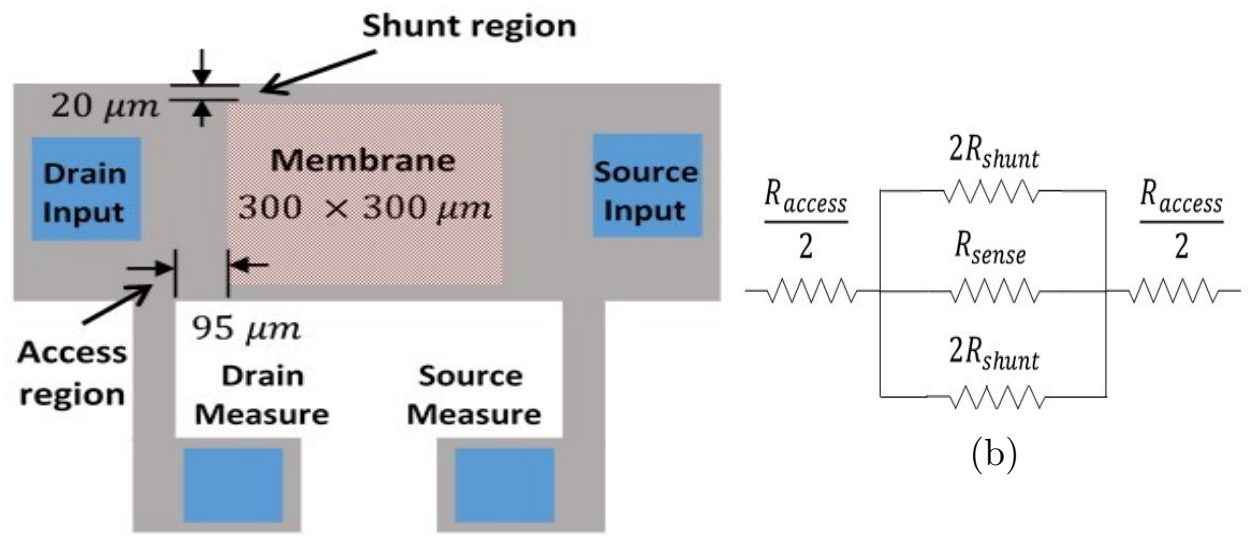

(a)

Figure 6: Schematics for (a) sensor layout with terminals (not to scale) (b) resistances including the parasitics between the measured terminals. 


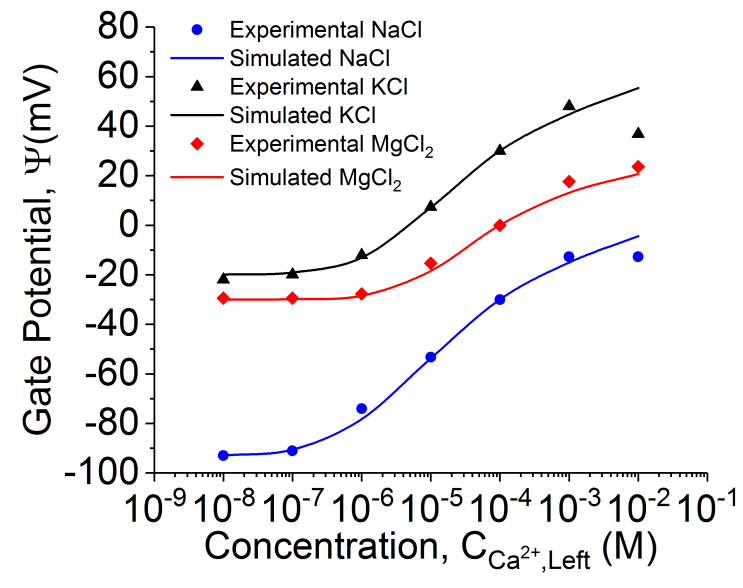

Figure 7: Experimental (dashed) and simulated (solid) response curves in $\mathrm{KCl}, \mathrm{MgCl}_{2}$, and $\mathrm{NaCl}$ solutions. 